\title{
Análisis de la prosodia semántica y su aplicación en la enseñanza del español a sinohablantes ${ }^{1}$
}

\section{Analysis of Semantic Prosody and its Application in Teaching Spanish to Chinese Speakers}

DOI: $10.32870 /$ mycp.v11i31.768

\author{
Yufei $\mathrm{Cao}^{2}$ \\ Shuqin Peng ${ }^{3}$
}

\begin{abstract}
Resumen
En el marco de la lingüística de corpus y con base en el Corpus del Español del Siglo XXI y el Corpus Nacional de Chino Contemporáneo, con el método propuesto por Zhao y Li (2010) y analizado mediante el programa AntConc, este trabajo realiza un análisis de la prosodia semántica con el caso ejemplificado del adverbio de grado español demasiado y su equivalente en chino 太(tài), asimismo, indaga en su aplicación didáctica. Las conclusiones del estudio son las siguientes: 1 . Tanto demasiado como 太(tài) cuentan con una prosodia semántica mixta y sus usos frecuentemente expresan una actitud negativa o neutral; 2. Al igual que太(tài), demasiado es usado como un tipo de eufemismo en oraciones negativas; 3. Demasiado en comparación con 太(tài) expresa en menos ocasiones una actitud positiva; 4 . Al impartir el español a sinohablantes, el estudio de la prosodia semántica puede contribuir a la profundización del análisis semántico de las palabras, visualizar sus combinaciones más frecuentes y evitar la transferencia negativa del chino.
\end{abstract}

Palabras clave: prosodia semántica, corpus, adverbio de grado, enseñanza del español, sinoha-

\begin{abstract}
In the framework of corpus linguistics and based on the Corpus of Spanish in the 21st Century and the Contemporary Chinese National Corpus, this paper takes Spanish adverb of degree demasiado ("too", in english) and its Chinese equivalent 太(tài) as example and analyses their semantic prosody with the method proposed by Zhao and Li (2010) and using the software AntConc; at the same time, it explores its teaching application. The conclusions of the study are as follows: 1 . Both demasiado and 太(tài) have mixed semantic prosody and their uses frequently express a negative or neutral attitude; 2. Same as 太(tài), demasiado is used as a kind of euphemism in negative sentences; 3 . Compared to 太(tài), demasiado expresses the positive attitude less; 4 . In the process of teaching Spanish to Chinese speakers, assisted by the study of semantic prosody we can deepen the semantic analysis of words, visualize their most common combinations and avoid the negative transfer of Chinese.
\end{abstract}

Keywords: semantic prosody, corpus, adverb of degree, Spanish teaching, Chinese speakers.

blantes.

Artículo recibido el 25 de abril de 2021 y dictaminado el 17 de agosto de 2021.

1. Agradecemos a los dos revisores anónimos sus comentarios y sugerencias, que han contribuido a mejorar la versión final de este trabajo. Este artículo es financiado por "Innovative Research Team of Shanghai International Studies University".

2. Shanghai International Studies University (sISU). C/Wenxiang Lu 1550, Shanghai, China, 201620. ORCID: https://orcid.org/ 0000-0003-0635-8110. Correo electrónico: yufeielisa@qq.com

3. Shanghai International Studies University (sisu). C/Wenxiang Lu 1550, Shanghai, China, 20162. ORCID: https://orcid.org/ 0000-0002-2245-0438. Correo electrónico: 929934413@qq.com 


\section{Introducción}

La prosodia semántica, un tema frecuentemente discutido en el ámbito de la lingüística de corpus, se refiere al "significado asociativo que sobrepasa el límite de las palabras". Para determinarla, es necesario utilizar el buscador de posicionamiento con el fin de averiguar las coligaciones de las palabras, sus combinaciones más frecuentes y analizar el significado asociativo tanto cuantitativa como cualitativamente con el apoyo de la tecnología de la informática moderna (Ji \& Wu, 2003). Stubbs (1996) clasifica la prosodia semántica en negativa, mixta y positiva. Entonces, si las palabras que estudiamos atraen en la mayoría de los casos combinaciones con actitud negativa, podemos afirmar que estas presentan una prosodia semántica negativa. Del mismo modo, se considera que las palabras conllevan un significado asociativo mixto si estas atraen al mismo tiempo cierta cantidad de combinaciones con actitudes positivas, neutrales y negativas (Wei, 2002).

Hoy en día, hay cada vez más estudiantes chinos que empiezan a estudiar español. A partir del año 2017 la lengua española comenzó a incorporarse en la enseñanza básica de China, y en el año 2020, cien universidades chinas ya contaban con el programa de pregrado en español y aproximadamente 20,000 o 22,000 estudiantes a nivel universitario estaban aprendiendo esta lengua (Zheng, 2020). A la hora de impartir el español a los alumnos chinos, ¿cómo profundizamos en el análisis semántico de las palabras y señalamos sus combinaciones más frecuentes? ¿Cómo visualizamos de manera vívida la enseñanza del léxico y evitamos al mismo tiempo la transferencia negativa de la lengua materna? Estas son las preguntas de partida de nuestro estudio y constituyen al mismo tiempo el objetivo de la investigación.

En la primera parte de este artículo se analizarán de manera breve los estudios relacionados con la prosodia semántica. Después, una vez efectuada la revisión bibliográfica, describiremos la metodología de investigación, analizaremos el adverbio de grado demasiado y discutiremos los resultados. Finalmente, indagaremos en las implicaciones pedagógicas y presentaremos las conclusiones más relevantes a las que llegó esta investigación.

\section{Estudios previos}

A partir de la década de 1980, la prosodia semántica comenzó a constituir el objeto de estudio de los lingüistas de diversas partes del mundo. Los primeros 
artículos publicados con respeto al tema están centrados en la lengua inglesa y estudian la interacción semántica que se da entre diferentes palabras combinadas (Sinclair, 1991; Louw, 1993). Otro tipo de estudios relacionados analizan las pequeñas diferencias que existen entre los sinónimos por medio del análisis cuantitativo de la prosodia semántica (You, 2011; Shen \& Yang, 2019).

A medida que avanza la lingüística de corpus en todo el mundo, los investigadores empiezan a realizar comparaciones entre las distintas lenguas. Por ejemplo, Zhao y Li (2010), Li y Wei (2012), Chen (2012) y Li (2013), basándose en los estudios de caso comparan la prosodia semántica entre el chino y el inglés. También, Berber (2000), Tognini-Bonelli (2002) y Tong y Xiu (2020), desde un marco de estudio contrastivo de la prosodia semántica comparan respectivamente el inglés y el portugués, el inglés y el italiano, así como el chino y el japonés. Este tipo de comparaciones siempre son muy interesantes, ya que permiten identificar sus aplicaciones en la traducción, la enseñanza de lenguas extranjeras y la elaboración de diccionarios. A pesar de que a lo largo de la revisión bibliográfica se encontraron numerosos trabajos comparativos, se halló que muy pocos están dedicados a la comparación de la prosodia semántica del español y el chino. Por lo tanto, este trabajo busca establecer un estudio comparativo de estas dos lenguas, y explorar a la vez su aplicación en la enseñanza del español a hablantes de chino.

\section{Metodología}

\subsection{Objeto de estudio}

Esta investigación toma como ejemplo típico del español el adverbio de grado demasiado y lo compara con su equivalente en chino 太(tài). Es importante señalar que este estudio se limita a investigar el uso de la palabra demasiado como adverbio de grado y no se analizan sus otros usos como adjetivo o pronombre, debido a que su análogo en chino 太(tài) pertenece únicamente a la clase de palabra adverbio. Al efectuar el análisis cualitativo, se consultó la última edición del Diccionario de la lengua española (2021) de la Real Academia Española (RAE), en el cual la palabra demasiado (adv.) se glosa como "excesivamente, a veces con un complemento introducido por la preposición 'para' que expresa una base de comparación". También puede indicar una intensidad que excede el límite conveniente o aceptable, una frecuencia excesiva o un tiempo excesivo. Según la séptima versión del Diccionario de la lengua china moderna 
del año 2016 (editado por la Academia China de Ciencias Sociales), el adverbio chino太(tài) significa excesivo en oraciones tanto positivas como negativas, sin embargo, solamente en las primeras manifiesta un alto nivel de elogio, mientras que en las segundas sirve para suavizar el tono y significa "muy".

En el caso que estudiamos, según la definición del diccionario de la RAE (2021), la palabra demasiado (adv.) significa "excesivamente", lo cual parece indicar que este adverbio cuenta con una prosodia semántica negativa. Sin embargo, como se mencionó en la introducción, el significado asociativo puede superar el límite de las palabras y, por consiguiente, cuando examinamos la prosodia semántica de una palabra, en lugar de analizarla de forma aislada debemos situarla en diversos textos, es decir, hemos de analizar la palabra junto con sus combinaciones y contextos (Stubbs, 1996). Por lo tanto, no podemos sacar una conclusión simplemente mediante la consulta del diccionario ni juzgar las palabras de manera aislada. En estos casos es necesario acudir al corpus para realizar un análisis cuantitativo, estudiar las palabras junto con sus combinaciones en diversos contextos y llegar así a una conclusión más precisa y clara.

\subsection{Corpus y datos}

En cuanto al análisis cuantitativo, se siguió el método más típico del estudio de la prosodia semántica propuesto por Zhao y Li (2010), y se utilizó la versión 0.92 del Corpus del Español del Siglo XXI (CORPES XXI) de la Real Academia Española (Real Academia Española, 2020) como objeto de observación. Este corpus cuenta con más de 300,000 documentos procedentes de textos escritos y de transcripciones orales originarias de España, América, Filipinas y Guinea Ecuatorial, con una distribución de 25 millones de formas por cada uno de los años comprendidos en el periodo de tiempo de 2001 a 2012. A diferencia del CREA (el Corpus de Referencia del Español Actual, una base de datos del español creada por la Real Academia Española), con el CORPES XXI es posible realizar búsquedas por lema, forma y clase de palabra. Por eso, para consultar la palabra demasiado como adverbio, se prefirió acudir al CORPES XXI.

En cuanto al corpus chino, se escogió el Corpus del Chino Moderno del Comité de Idiomas de China (CNCORPUS) como objeto de observación (Ministerio de Educación, 2020). El CNCORPUS es el corpus más equilibrado del chino moderno con una amplia gama de materiales y un gran lapso de tiempo. Adicionalmente, está formado por textos procedentes de manuales, periódi- 
cos, publicaciones integrales, publicaciones profesionales, libros, entre otros. Contiene cerca de 20 millones de caracteres chinos y los datos proporcionados para la búsqueda en línea están sujetos a la segmentación de palabras al igual que a la anotación de clase de palabra. Por eso, es posible buscar por palabra con clase de palabra determinada.

Con el objetivo de que el estudio sea más representativo e indique mayor universalidad, al buscar el adverbio demasiado en el CORPES XXI, no filtramos el país ni el tipo de lengua, mientras que al consultar su equivalente chino 太 (tài), los datos buscados pertenecen a la lengua escrita porque los materiales del CNCORPUS son textos escritos. Entonces, resulta bastante conveniente ya que se pueden desarrollar futuras investigaciones filtrando las condiciones de consulta según el objetivo de estudio. Por ejemplo: es posible examinar si existen diferencias en la prosodia semántica de alguna palabra española entre la lengua oral y la escrita o entre los dialectos de los diferentes países.

\subsection{Herramientas y procesamiento de datos}

La principal herramienta empleada para el procesamiento de los datos descargados de los corpus es el software AntConc versión 3.5.8 (Anthony, 2020), una herramienta gratuita y multiplataforma que se utiliza para el aprendizaje basado en datos y para llevar a cabo investigaciones que se fundamentan en la lingüística de corpus. A través de su Concordance Tool o función de concordancias, se muestran los resultados de búsqueda en formato KWIC (palabras clave en contexto, por sus siglas en inglés), permitiendo observar cómo se usan las palabras y frases frecuentemente en un corpus de textos. La función Clusters muestra grupos de palabras según los criterios de búsqueda y resume los resultados generados en las funciones Concordance o Concordance Plot. La función Collocates o Coligaciones se utiliza para generar una lista ordenada de las coligaciones que aparecen junto al término buscado, permitiendo encontrar patrones no secuenciales en el lenguaje. Aún más, la función Word List o Lista de palabras cuenta todas las palabras del corpus y las presenta en una lista ordenada, dando la posibilidad de encontrar rápidamente las palabras más frecuentes en un corpus. ${ }^{4}$ Además de esta herramienta, el programa Tree Tagger 3.0 (Schmid, 2019) fue usado para la anotación de la clase de palabra a los datos brutos con el fin de procesarlos con facilidad en la herramienta

4. https://www.laurenceanthony.net/software/antconc/resources/help_AntConc344_spanish.pdf 
AntConc. Adicionalmente, elegimos EmEditor Profesional 12.0.0 (Emurasoft, Inc., 2012) para editar el texto y analizar claramente los resultados hallados en AntConc.

A grandes rasgos el proceso consistió en que una vez las herramientas anteriormente mencionadas estaban listas, se buscó primero en el CORPES XXI todas las unidades léxicas en que aparece la palabra demasiado. En este corpus se encontraron en total 39,555 unidades que contienen la palabra demasiado, dentro de las cuales descargamos de forma aleatoria 1,083 como muestra de estudio. ${ }^{5}$ Después de la anotación de la muestra de estudio, se extrajeron todas las unidades en que la palabra demasiado funciona como adverbio de grado a través de la Concordance Tool del software AntConc. Seguidamente, se eliminaron algunas unidades repetitivas y tras efectuar esta operación quedaron 992 unidades léxicas. Mediante la observación de estas unidades, encontramos la colligation o coligación de la palabra demasiado (adv.). La coligación determina la relación entre las categorías gramaticales en el texto, por lo que es un concepto muy importante en el estudio de la combinación de palabras. Se refiere a la unidad léxica y a la estructura gramatical en que aparece la combinación de palabras (Wei, 2002). Después de este paso y con el propósito de estudiar los casos más representativos, se extrajeron 487 unidades en las que aparecen las palabras que con mayor frecuencia (más de dos veces) se combinan con demasiado, para ello se usó la función Collocates. Por último, estudiamos la palabra demasiado en estas 487 unidades extraídas junto con sus respectivos contextos, las cuales se clasificaron según sus actitudes expresadas (positiva, negativa o neutral) y así se llegó a una conclusión sobre la prosodia semántica del adverbio demasiado.

De igual manera se llevó a cabo la búsqueda y el procesamiento de los datos del adverbio de grado chino 太(tài). Cabe señalar que se analizaron 992 unidades léxicas que fueron seleccionadas de forma aleatoria entre todos los 2,942 resultados encontrados en el CNCORPUS a fin de mantener la uniformidad de número de unidades analizadas con el adverbio demasiado.

\section{Análisis de resultados}

A partir de la observación de esta muestra de estudio se puede afirmar que las coligaciones más prototípicas de la palabra demasiado son las siguientes:

5. 1,083 es el número que representa una descarga automática de forma aleatoria en el CORPES XXI. 
1. V.+ADV., por ejemplo, "Usted habla demasiado. ¿No sabe hacer otra cosa con la boquita?"; 2. ADV.+ADJ., por ejemplo, “... la desigualdad de fuerzas entre los yanquis y nosotros es demasiado grande;...”; 3. ADV.+ADV., por ejemplo, “... aquí afuera las cosas no andan demasiado bien, mientras más rápido se muere menos se sufre...".

Este análisis de la prosodia semántica se desarrolla dentro de las coligaciones anteriormente mencionadas y teniendo en cuenta que los verbos de combinación están a la izquierda de la palabra clave demasiado, mientras que los adjetivos y los adverbios se encuentran a la derecha, realizamos el análisis en dos pasos.

En primer lugar, se extraen de la coligación "V.+ADV." todos los verbos que se encuentran a través de la función Concordance de AntConc. Los verbos encontrados se clasificaron en dos tipos: el primero corresponde a los verbos no copulativos directamente modificados por el adverbio demasiado, cuya actitud en combinación con demasiado puede ser juzgada con base en el mismo verbo junto con sus contextos. El otro tipo son los verbos copulativos ser, estar y parecer, y los verbos que desempeñan una función semejante, tales como; quedar, sentir (se), encontrar (se), sonar, poner (se), mostrar (se), resultar, etc. Por ejemplo, "Ese padre estaba demasiado gordo", "A Consuelo le parece demasiado frágil y joven como para ser madre", "Te digo: es demasiado triste la vida del viajero".

Como muestran los ejemplos anteriormente citados, en este tipo de combinación, la palabra demasiado no modifica directamente a los verbos sino modifica a los adjetivos o adverbios. Es decir, en estos casos la actitud expresada por la unidad léxica con demasiado depende de los adjetivos o adverbios y, por lo tanto, en lugar de analizar estos verbos copulativos (estaba, parece, es, etcétera), tenemos que examinar los adjetivos o adverbios que se encuentran a la derecha de la palabra demasiado (demasiado gordo, demasiado frágil y joven, demasiado triste). En este paso de análisis estadístico y al eliminar el segundo tipo de verbos, obtenemos 142 unidades léxicas con verbos no copulativos modificados directamente por demasiado y las enumeramos en la siguiente tabla. 
Tabla 1

Verbos que más frecuentemente se combinan con demasiado

\begin{tabular}{c|c|c|c|c|c}
\hline Verbo & Frecuencia & Verbo & Frecuencia & Verbo & Frecuencia \\
\hline hablar & 15 & molestar & 3 & entender & 2 \\
\hline importar & 9 & levantar & 3 & detener & 2 \\
\hline exigir & 9 & gustar & 3 & descender & 2 \\
\hline beber & 9 & esperar & 3 & creer & 2 \\
\hline preocupar & 7 & esforzar & 3 & conocer & 2 \\
\hline tardar & 6 & comer & 3 & caminar & 2 \\
\hline saber & 4 & aproximar & 3 & cambiar & 2 \\
\hline pesar & 4 & alardear & 3 & aumentar & 2 \\
\hline pedir & 4 & sufrir & 2 & arriesgar & 2 \\
\hline acercar & 4 & sorprender & 2 & apreciar & 2 \\
\hline variar & 3 & recargar & 2 & amar & 2 \\
\hline trabajar & 3 & quejarse & 2 & alejar & 2 \\
\hline pensar & 3 & interesar & 2 & mover & 2 \\
\hline & & & & gritar & 2 \\
\hline
\end{tabular}

Fuente: Elaboración propia.

Al efectuar el análisis de las actitudes de los casos presentados en la tabla 1 dentro del marco de la coligación "V.+ADV." se identificó que los verbos con significado desfavorable, tales como beber, exigir, tardar, etc., cuando se hallan en oraciones afirmativas, su combinación con el adverbio demasiado destaca aún más la actitud negativa. Por ejemplo, en la oración "Ese hombre bebe demasiado, es un alcohólico, probablemente sea un violento", la combinación de bebe con demasiado expresa "adicto a la bebida alcohólica", lo cual indica una actitud negativa.

Por otro lado, hay otros verbos que, aunque cuentan con un significado favorable, tales como importar, trabajar, entre otros, implican "un grado excesivo" cuando se combinan con demasiado, y por lo tanto también expresan una actitud negativa. Por ejemplo: la palabra importar significa "interesar" y por eso decimos que cuenta con un significado favorable. Sin embargo, cuando analizamos esta palabra en diferentes contextos, obtenemos resultados distintos. Entonces, si analizamos la oración "Dijo que uno nunca llegaba a conocer a nadie de verdad. Que cuando alguien le importaba demasiado, volvía a sentir el mismo miedo. El miedo a que la dejaran sola", considerando el contexto, sobre todo la palabra miedo, podemos afirmar que "importar demasiado" 
significa "importar excesivamente", lo cual puede causar miedo. Por lo tanto, se trata de un caso que expresa una actitud negativa. En otros contextos, la misma combinación no muestra ni actitud positiva ni negativa. De ese modo en "No sé quién lo preguntó. Tampoco importaba demasiado. Me di cuenta de que también yo, desde que había leído el anuncio en el diario, lo había imaginado así: [...]" la combinación "importar demasiado" es simplemente una afirmación y por lo cual consideramos que se trata de una actitud neutral.

Por medio de los pasos descritos anteriormente, clasificamos las actitudes expresadas por estos 142 casos en positivas, negativas y neutrales. El resultado de la clasificación puede consultarse en la tabla 2.

\section{Tabla 2}

Actitudes expresadas por "V. + demasiado"

\begin{tabular}{c|c|c|c}
\hline Actitud expresada por "V. + demasiado" & Positiva & Negativa & Neutral \\
\hline Frecuencia & 8 & 57 & 77 \\
\hline Porcentaje & $5.6 \%$ & $40.2 \%$ & $54.2 \%$ \\
\hline
\end{tabular}

Fuente: Elaboración propia.

En la tabla 2 se puede observar que en la mayoría de los casos (más del $50 \%$ ) la actitud expresada por la combinación "verbo no copulativo + demasiado" es neutral. Eso se debe a dos razones: por un lado, muchos de los verbos que más frecuentemente se combinan con demasiado son de significado neutral, tales como hablar, saber y conocer. Por otro lado, muchos verbos con significado desfavorable aparecen en oraciones negativas en que la negación expresada por tampoco, no, sin, nunca resta la actitud negativa y la convierten en neutral e incluso positiva. Es decir, las actitudes neutrales e incluso positivas expresadas por la coligación "verbo no copulativo + demasiado" se deben en parte al hecho de que estas combinaciones a menudo aparecen en oraciones negativas, y la negación no solo puede transformar palabras con significado desfavorable en unidades léxicas con actitudes neutrales e incluso positivas, sino que también puede reducir el grado positivo de las palabras con significado favorable, convirtiéndolas en unidades que expresan actitudes neutrales.

Veamos algunos ejemplos concretos. La palabra quejarse en "Greiz no podía quejarse demasiado de AYW y AYW no podía quejarse demasiado de Greiz. Una situación bastante envidiable...", al combinarse con el adverbio negativo no, pierde su propio significado desfavorable y muestra una actitud positiva, 
ya que en este caso se evidencia una relación armoniosa en la cual "las dos personas no se quejan mutuamente". Otro ejemplo representativo es preocuparse, un verbo que cuenta con un significado desfavorable. Sin embargo, al ser insertado en la oración "Si en el blues el hombre se resignaba a su destino, en el góspel se lo agradecía al Señor, en el country cantaba al presente sin preocuparse demasiado por el pasado y en el folk dejaba testimonio de su disconformidad, en el rock se rebeló", "sin preocuparse demasiado" muestra una actitud bastante optimista de "dejar atrás el pasado" gracias a la aparición del adverbio negativo sin.

Es importante aclarar que el hecho de que la negación pueda neutralizar las actitudes expresadas no significa que lo haga en todas las situaciones. A veces, los adverbios negativos también pueden destacar la actitud negativa e incluso el sentido irónico. Por ejemplo, en la oración "El comienzo de estas manifestaciones armadas en ambiente rural, especialmente las llevadas a cabo de 1959 hasta fines de la década del 60, no preocuparon demasiado a gran parte de la sociedad argentina y al mismo gobierno de turno (constitucional o de facto)... la verdadera tragedia que la amenazaba...", la combinación de no y preocupar demasiado resalta que el gobierno argentino y su población no son conscientes de la grave crisis con que se enfrentan, y por lo tanto muestra de manera irónica una actitud negativa.

El segundo paso consistió en extraer de las coligaciones "ADV. +ADJ." y "ADV. +ADV." los adjetivos y los adverbios que más frecuentemente se combinan con demasiado (con una aparición de más de dos veces) a través de la función Collocates de AntConc. Tras realizar esa operación, se obtuvieron 345 unidades léxicas en las que se encuentran en total 63 adjetivos y adverbios, tales como tarde, grande, lejos, fuerte, bueno, pronto, entre otros; véase tabla 3. 
Tabla 3

Adjetivos y adverbios que más frecuentemente se combinan con demasiado

\begin{tabular}{c|c|c|c|c|c}
\hline Palabra & Frecuencia & Palabra & Frecuencia & Palabra & Frecuencia \\
\hline tarde & 49 & caro & 5 & seco & 2 \\
\hline grande & 32 & seguro & 4 & radical & 2 \\
\hline lejos & 22 & breve & 4 & pretencioso & 2 \\
\hline fuerte & 13 & joven & 4 & presente & 2 \\
\hline viejo & 12 & inteligente & 4 & pobre & 2 \\
\hline bien & 12 & claro & 4 & numeroso & 2 \\
\hline largo & 11 & profundo & 3 & junto & 2 \\
\hline cerca & 10 & simple & 3 & intenso & 2 \\
\hline bueno & 8 & lejano & 3 & insignificante & 2 \\
\hline rico & 7 & humano & 3 & independiente & 2 \\
\hline pronto & 7 & fantasioso & 3 & impreciso & 2 \\
\hline peligroso & 7 & estrecho & 3 & imaginativo & 2 \\
\hline temprano & 6 & directo & 3 & hermoso & 2 \\
\hline perfecto & 6 & bajo & 3 & grueso & 2 \\
\hline alto & 6 & ancho & 3 & flaco & 2 \\
\hline lento & 5 & amplio & 3 & extenso & 2 \\
\hline importante & 5 & vivo & 2 & efusivo & 2 \\
\hline gordo & 5 & visible & 2 & cobarde & 2 \\
\hline formal & 5 & violento & 2 & barato & 2 \\
\hline evidente & 5 & triste & 2 & ambicioso & 2 \\
\hline duro & 5 & sucio & 2 & absurdo & 2 \\
\hline
\end{tabular}

Fuente: Elaboración propia.

Para explicar las coligaciones "ADV. +ADJ." y "ADV. +ADV." de la palabra demasiado, se puede acudir a la Ciyunwenzi (Ciyun Ltd., 2020) para así trazar una nube de palabras (figura 1) basada en los adjetivos y los adverbios que con más frecuencia se combinan con demasiado extraídos por AntConc. 
Figura 1

Nube de palabras

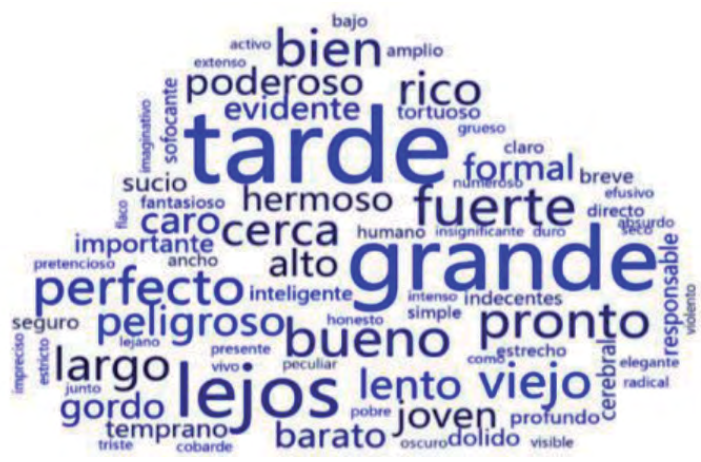

Fuente: Elaboración propia con Ciyunwenzi (Ciyun Ltd., 2020).

Esta figura muestra claramente que los adjetivos y los adverbios que más frecuentemente acompañan a demasiado son tarde, grande, lejos, fuerte, bueno, pronto, y así sucesivamente. Además, el tamaño de la letra indica visualmente el grado de frecuencia de combinación. Entonces, consideramos que en el proceso de enseñanza de español a los estudiantes sinohablantes, tales imágenes auxiliares son vívidas e impresionantes, lo cual puede permitir que aprendan más rápidamente las combinaciones de las palabras.

Analizamos respectivamente estas 345 unidades léxicas en que aparece la coligación "demasiado + ADJ. o ADV." y efectuamos la clasificación según la actitud expresada. Por ejemplo, la palabra grande es un adjetivo de significado neutral que se puede analizar de acuerdo a los contextos específicos en que se halla, como se muestra a continuación: "la desigualdad de fuerzas entre los yanquis y nosotros es demasiado grande; ellos pueden exterminarnos sin arriesgar sus vidas...", la palabra desigual implica que la combinación "demasiado grande" expresa una actitud negativa.

En cuanto al caso "Lo que ella sentía entonces era indescriptible y superior a los sentidos y demasiado grande para compartirlo. Cada uno de esos encuentros le recordaba la salida del sol o cuando amainaba un aguacero y todos los verdes del mundo quedaban brillando", considerando el contexto y las imágenes "la salida del sol", "amainaba un aguacero" y "los verdes brillan", juzgamos que se trata de un caso con actitud positiva que expresa "un sentido de felicidad indescriptible". Otro ejemplo del corpus es "También sería 
importante que la brecha educacional entre los diferentes públicos no fuera demasiado grande y que aquellos tuvieran un poder adquisitivo similar en cuanto al acceso y a la compra de productos informativos...", la combinación de no y demasiado convierte la actitud negativa que expresa "brecha educacional + grande" en una actitud neutral, lo cual sirve para suavizar el tono por parte del emisor. Por lo tanto, este caso se categorizó como "neutral".

Mediante los parámetros de análisis arriba mencionados, exponemos los 345 casos (demasiado + ADJ. o ADV.) en la tabla 4.

\section{Tabla 4}

Actitudes expresadas por "demasiado + adj. o adv."

\begin{tabular}{c|c|c|c}
\hline Actitud expresada por "demasiado + ADJ. o ADV." & Positiva & Negativa & Neutral \\
\hline Frecuencia & 40 & 177 & 128 \\
\hline Porcentaje & $11.6 \%$ & $51.3 \%$ & $37.1 \%$ \\
\hline
\end{tabular}

Fuente: Elaboración propia.

En la tabla 5 se sintetizan todos los casos de las tres coligaciones "V. + ADV.", "ADV. + ADJ." Y "ADV. + ADV."

\section{Tabla 5}

Actitudes expresadas por demasiado (adv.)

\begin{tabular}{c|c|c|c}
\hline Actitud expresada por demasiado (adv.) & Positiva & Negativa & Neutral \\
\hline Frecuencia & 48 & 234 & 205 \\
\hline Porcentaje & $9.8 \%$ & $48.1 \%$ & $42.1 \%$ \\
\hline
\end{tabular}

Fuente: Elaboración propia.

Como se evidencia en la tabla 5, el resultado no apoya que el adverbio demasiado tenga una prosodia semántica negativa, ya que, aunque los casos con actitud negativa presentan una tendencia bastante alta, el porcentaje de los casos con actitud neutral y positiva es del $51.9 \%$, lo cual supera la mitad de los casos. En otras palabras, aproximadamente un $48 \%$ de las combinaciones de demasiado expresan una actitud negativa, mientras que un $51,9 \%$ expresan una actitud positiva o neutral. Por lo tanto, el adverbio demasiado tiene una prosodia semántica mixta. 
En este mismo orden de ideas y sobre la base de la observación de la muestra de estudio, se puede afirmar que las coligaciones más prototípicas de la palabra 太(tài) son las siguientes: 1. ADV. +V., por ejemplo, “相比之下, 我们过去太缺乏冒险精神了。” (En cambio, carecemos excesivamente de espíritu aventurero.); 2. ADV. +ADJ., por ejemplo, “不行, 这个办法显然太 蚌。” (No, este método es obviamente demasiado estúpido.); 3. ADV. +ADV., por ejemplo, “这次大地震来得太突然, 发电厂和供电线路都遭到了严重破 坏。” (El terremoto se produjo de forma demasiado repentina. Las centrales eléctricas y las líneas de suministro eléctrico sufrieron graves daños.)

El análisis de la prosodia semántica de 太(tài) también se desarrolla dentro de las coligaciones presentadas anteriormente, sin embargo, en este caso todos los verbos, adjetivos y adverbios de combinación están a la derecha de la palabra clave 太(tài). A través de la función Collocates de AntConc a condición del span (distancia a la palabra clave) 0-2R (incluidas las dos palabras más cercanas a la derecha de 太[tài]) fue posible obtener las 134 palabras que más frecuentemente se combinan con 太(tài) (con una aparición de más de dos veces) entre las 784 unidades léxicas del corpus, tales como grande, bueno, lejos, pequeño, entre otras que se señalan en la tabla 6 .

Tabla 6

Palabras que más frecuentemente se combinan con 太(tài)

\begin{tabular}{l|c|l|c|l|c}
\hline Palabra & Frecuencia & Palabra & Frecuencia & Palabra & Frecuencia \\
\hline 大 (grande) & 66 & 热 (caliente) & 4 & $\begin{array}{l}\text { 过火 } \\
\text { (exagerado) }\end{array}$ & 2 \\
\hline 好 (bueno) & 47 & 漂亮 (hermoso) & 4 & $\begin{array}{l}\text { 轻信 (creer a la } \\
\text { ligera) }\end{array}$ & 2 \\
\hline 不 (no) & 43 & 浓 (denso) & 4 & 软 (blando) & 2 \\
\hline 少 (poco) & 35 & 没有 (no tener) & 4 & 讲究(cuidadoso) & 2 \\
\hline $\begin{array}{l}\text { 远 (lejano, } \\
\text { lejos) }\end{array}$ & 29 & 惨 (trágico) & 4 & 被动 (pasivo) & 2 \\
\hline $\begin{array}{l}\text { 小 (pequeño) } \\
\text { 快 (rápido) }\end{array}$ & 27 & 弱 (débil) & 4 & 薄 (delgado) & 2 \\
\hline 高 (alto) & 20 & $\begin{array}{l}\text { 年轻 (joven) } \\
\text { pena) }\end{array}$ & 4 & 艰难 (arduo) & 2 \\
\hline 长 (largo) & 17 & 严格 (estricto) & 4 & $\begin{array}{l}\text { 脆弱 (débil y } \\
\text { frágil) }\end{array}$ & 2 \\
\hline 重 (pesado) & 13 & 不好 (no bueno) & 4 & 脆 (quebradizo) & 2 \\
\hline
\end{tabular}


Análisis de la prosodia semántica y su aplicación en la enseñanza del español a sinohablantes

\begin{tabular}{|c|c|c|c|c|c|}
\hline Palabra & Frecuencia & Palabra & Frecuencia & Palabra & Frecuencia \\
\hline 近 (cercano) & 13 & $\begin{array}{l}\text { 不够 } \\
\text { (insuficiente) }\end{array}$ & 4 & 粗俗 (vulgar) & 2 \\
\hline 低 (bajo) & 12 & 饱 (harto) & 3 & 简单 (fácil) & 2 \\
\hline $\begin{array}{l}\text { 久 (de larga } \\
\text { duración) }\end{array}$ & 12 & 遥远 (lejano) & 3 & 笨 (estúpido) & 2 \\
\hline 短 (corto) & 11 & 迟 (tarde) & 3 & 稀罕 (apreciar) & 2 \\
\hline 深 (profundo) & 11 & 过分 (excesivo) & 3 & 矮 (bajo) & 2 \\
\hline 慢 (lento) & 11 & $\begin{array}{l}\text { 过于 (con } \\
\text { exceso) }\end{array}$ & 3 & $\begin{array}{l}\text { 看不起 } \\
\text { (despreciar) }\end{array}$ & 2 \\
\hline 强 (fuerte) & 9 & $\begin{array}{l}\text { 过 } \\
\text { (excesivamente) }\end{array}$ & 3 & 犯 (cometer) & 2 \\
\hline 紧 (tenso) & 8 & 认真 (aplicado) & 3 & 熟 (familiar) & 2 \\
\hline 差 (mal) & 8 & 荒唐 (absurdo) & 3 & 满 (lleno) & 2 \\
\hline $\begin{array}{l}\text { 危险 } \\
\text { (peligroso) }\end{array}$ & 8 & 老实 (honrado) & 3 & 湿 (húmedo) & 2 \\
\hline 贵 (caro) & 7 & 老 (viejo) & 3 & 淡 (soso) & 2 \\
\hline $\begin{array}{l}\text { 突然 } \\
\text { (repentino) }\end{array}$ & 7 & $\begin{array}{l}\text { 短暂 } \\
\text { (transitorio) }\end{array}$ & 3 & $\begin{array}{l}\text { 枯燥 } \\
\text { (monótono) }\end{array}$ & 2 \\
\hline 早 (temprano) & 7 & 盛 (vigoroso) & 3 & 机械 (mecánico) & 2 \\
\hline 厉害 (fuerte) & 7 & 疲倦 (cansado) & 3 & $\begin{array}{l}\text { 有意思 } \\
\text { (interesante) }\end{array}$ & 2 \\
\hline $\begin{array}{l}\text { 辛苦 } \\
\text { (laborioso) }\end{array}$ & 6 & 狠 (cruel) & 3 & 明显 (evidente) & 2 \\
\hline 轻 (ligero) & 6 & 浪费 (malgastar) & 3 & 无聊 (aburrido) & 2 \\
\hline 死 (inflexible) & 6 & 棒 (excelente) & 3 & 敏感 (sensible) & 2 \\
\hline $\begin{array}{l}\text { 感谢 } \\
\text { (agradecer) }\end{array}$ & 6 & 晚 (tarde) & 3 & 放肆 (insolente) & 2 \\
\hline 容易 (fácil) & 6 & 无 (no) & 3 & 挤 (apretado) & 2 \\
\hline 好了(bien) & 6 & 广 (amplio) & 3 & 弯曲 (corvado) & 2 \\
\hline 坏 (malo) & 6 & 奇怪 (raro) & 3 & 平常 (normal) & 2 \\
\hline 苦 (amargo) & 5 & 天真 (inocente) & 3 & $\begin{array}{l}\text { 巧 (por } \\
\text { casualidad) }\end{array}$ & 2 \\
\hline 爱 (amar) & 5 & 多 (mucho) & 3 & $\begin{array}{l}\text { 小看 } \\
\text { (menospreciar) }\end{array}$ & 2 \\
\hline 浅 (somero) & 5 & 可惜 (penoso) & 3 & 复杂 (complejo) & 2 \\
\hline 急 (urgente) & 5 & 可怜 (pobre) & 3 & 喜欢 (querer) & 2 \\
\hline 忙 (ocupado) & 5 & 厚 (grueso) & 3 & 可爱 (simpático) & 2 \\
\hline 客气 (amable) & 5 & 劳累 (agotado) & 3 & 值得 (merecer) & 2 \\
\hline 可怕 (terrible) & 5 & 冷 (frío) & 3 & 冷酷 (despiado) & 2 \\
\hline
\end{tabular}




\begin{tabular}{|c|c|c|c|c|c|}
\hline Palabra & Frecuencia & Palabra & Frecuencia & Palabra & Frecuencia \\
\hline $\begin{array}{l}\text { 不象话 } \\
\text { (irrazonable) }\end{array}$ & 5 & $\begin{array}{l}\text { 兴奋 } \\
\text { (emocionado) }\end{array}$ & 3 & $\begin{array}{l}\text { 冒险 } \\
\text { (arriesgado) }\end{array}$ & 2 \\
\hline 难 (difícil) & 4 & 俊 (tonto) & 3 & $\begin{array}{l}\text { 保守 } \\
\text { (conservador) }\end{array}$ & 2 \\
\hline 美 (bonito) & 4 & 麻烦 (molestar) & 2 & 令 (hacer) & 2 \\
\hline $\begin{array}{l}\text { 缺乏 } \\
\text { (escasear) }\end{array}$ & 4 & 高兴 (alegre) & 2 & 严重 (grave) & 2 \\
\hline 累 (cansado) & 4 & 露 (expuesto) & 2 & $\begin{array}{l}\text { 不方便 } \\
\text { (inconveniente) }\end{array}$ & 2 \\
\hline $\begin{array}{l}\text { 粗心 } \\
\text { (negligente) }\end{array}$ & 4 & $\begin{array}{l}\text { 难堪 } \\
\text { (embarazoso) }\end{array}$ & 2 & $\begin{array}{l}\text { 不成话 } \\
\text { (irrazonable) }\end{array}$ & 2 \\
\hline $\begin{array}{l}\text { 狂妄 } \\
\text { (soberbio) }\end{array}$ & 4 & 小气 (tacaño) & 2 & & \\
\hline
\end{tabular}

Fuente: Elaboración propia.

Con el mismo método de análisis, en la tabla 7 resumimos las actitudes positivas, negativas o neutrales halladas en el corpus.

\section{Tabla 7}

Actitudes expresadas por 太(tài)

\begin{tabular}{c|c|c|c}
\hline Actitud expresada por “太(tài)” & Positiva & Negativa & Neutral \\
\hline Frecuencia & 107 & 476 & 201 \\
\hline Porcentaje & $13.7 \%$ & $60.7 \%$ & $25.6 \%$ \\
\hline
\end{tabular}

Fuente: Elaboración propia.

Según los resultados, el adverbio 太(tài) presenta una prosodia semántica mixta, pues, aunque los casos que expresan una actitud negativa corresponden al $60.7 \%$, el porcentaje de los casos con actitud neutral y positiva es de una porción de más de un tercio, es decir, uno de los tres 太(tài) no está en la prosodia semántica negativa, por lo que no se puede considerar simplemente que太(tài) tiene una prosodia semántica negativa.

\section{Comparación y aplicación didáctica}

La investigación de la prosodia semántica de la palabra demasiado y el estudio comparativo con su equivalente chino 太(tài) puede contribuir a la profun- 
dización del análisis semántico de palabra, al igual que permite reducir la transferencia negativa de la lengua materna en la aplicación didáctica.

Figura 2

Prosodia semántica de demasiado y太(tài)

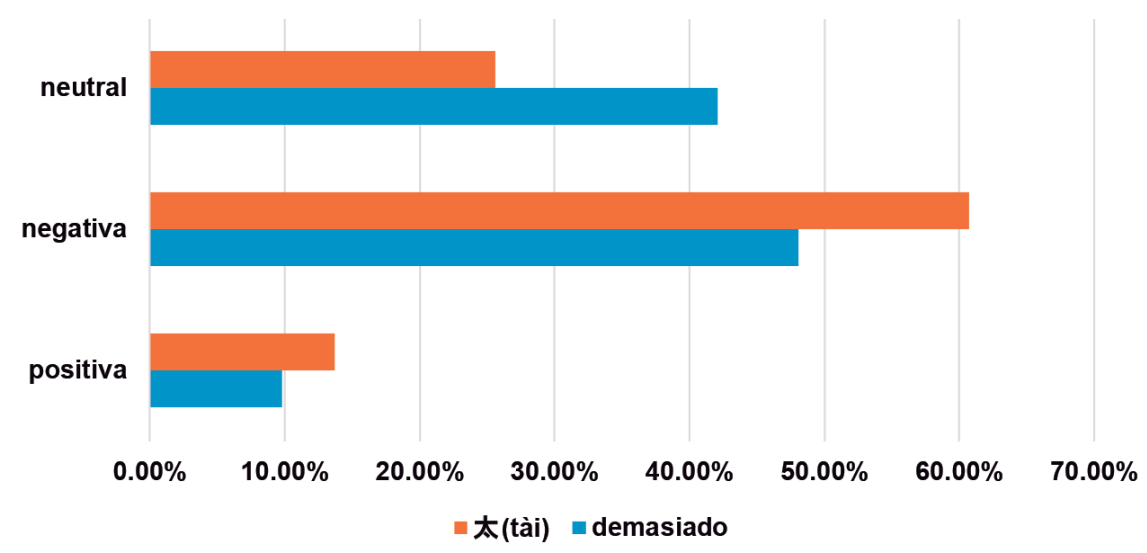

Fuente: Elaboración propia.

En primer lugar, mediante la figura 2 podemos constatar que el porcentaje de los casos de actitud positiva del adverbio chino 太(tài) (13.7 \%) es mayor que el de demasiado (9.8\%). Es decir, el adverbio demasiado expresa en menos ocasiones la actitud positiva en comparación con 太(tài). Por otro lado, como se ha explicado anteriormente, la aparición de demasiado suele ir acompañada de estructuras negativas, lo cual representa de hecho un empleo eufemístico para suavizar el tono y expresa una actitud neutral. Además, en las pocas oraciones afirmativas que encontramos, la combinación de demasiado con palabras de significado favorable a menudo tiene interpretación irónica al ser insertada en determinados contextos. Por ejemplo, en el CORPES XXI encontramos una oración en la que el adverbio demasiado se combina con la palabra de significado favorable hermoso: "Eres demasiado joven y demasiado hermosa. Y ahora hasta eres rica. Tarde o temprano reconstruirás tu vida (caramba, esto lo estoy escribiendo yo, iprecisamente yo! Pero ahora no tengo tiempo para pensar mejor las palabras). Quiero decir, carajo, que me reventaría que perdieras más de un día llorando por mí". Sin embargo, en este caso la expresión "demasiado hermosa" no es una simple alabanza sino más bien una excusa para expresar que "a pesar de su belleza, la chica no es adecuada para el chico 
que emite esta oración". Por lo tanto, cuando se quiere expresar actitudes positivas tales como el elogio, el empleo del demasiado no es muy apropiado.

Además, al efectuar el análisis del corpus, descubrimos que en los casos de actitud positiva los adverbios o adjetivos con significado favorable que admite demasiado en español son muy limitados (bien, bueno, etcétera), mientras que los que pueden combinarse con 太(tài) en chino son más variados, tales como hermoso, bonito, alegre y simpático, entre otros. Por ejemplo, “她实在太漂亮了” (ella + realmente + demasiado + hermosa + partícula de afirmación), “战士们实 在太可爱了” (los soldados + realmente + demasiado + simpáticos + partícula de afirmación), “飞机实在太美了” (los aviones + realmente + demasiado + bonitos + partícula de afirmación), “他太高兴了” (él + demasiado + alegre + partícula de afirmación)". Por lo tanto, a la hora de enseñar demasiado a los alumnos chinos, resulta esencial señalar esta diferencia entre el chino y el español para así procurar que los estudiantes eviten los usos inadecuados generados por la transferencia negativa del chino, tales como "\#Ella es demasiado hermosa", "\#Los soldados son demasiado simpáticos", "\#Los aviones son demasiado bonitos", "\#Él está demasiado alegre". ${ }^{6}$

En segundo lugar, a pesar de la aparente prosodia negativa que indica el diccionario español (RAE, 2021), este análisis muestra que el adverbio demasiado cuenta de hecho con una prosodia semántica neutral más marcada que en el caso del adverbio chino 太(tài). Esto se debe a que los verbos que con más frecuencia se combinan con demasiado no representan significado ni favorable ni desfavorable, es decir, estos verbos suelen explicar o describir un hecho de manera objetiva sin mostrar una actitud obvia. De igual manera, el análisis del corpus pone en evidencia que la palabra demasiado se utiliza a menudo en las oraciones negativas en que la negación (tampoco, no, sin, nunca, etcétera) puede quitar cierto grado de negatividad del adverbio demasiado y de esta forma permite una interpretación neutral e incluso positiva.

Adicionalmente, con una observación más detallada, hemos descubierto que el adverbio demasiado puede suavizar el tono en las oraciones negativas, como se indica en el ejemplo "También sería importante que la brecha educacional entre los diferentes públicos no fuera demasiado grande y que aquellos tuvieran un poder adquisitivo similar en cuanto al acceso y a la compra de productos informativos...". Este caso trata de un tipo de eufemismo (es decir, manifestación suave o decorosa de ideas cuya directa y franca expresión sería

6. El signo \# indica usos inadecuados. 
dura o malsonante) que expresa la palabra demasiado en el empleo negativo. De hecho, aunque este empleo eufemístico no se menciona en el Diccionario de la RAE, constituye un uso que comparte demasiado con su contraparte en chino 太(tài). Por lo tanto, cuando explicamos la palabra demasiado a los hablantes de chino, aparte de explicar sus sentidos básicos glosados en el diccionario, podemos ilustrar su función de suavizar el tono con unos ejemplos sacados del corpus, y de esta manera lograremos profundizar la explicación semántica del vocabulario y aprovechar la transferencia positiva de la lengua materna de los alumnos chinos a fin de mejorar la eficiencia de la enseñanza.

\section{Conclusiones}

Basado en los datos del corpus y mediante el software de AntConc y otras herramientas auxiliares, este artículo examina la prosodia semántica del adverbio de grado demasiado. Nuestro análisis muestra que el adverbio demasiado cuenta con una prosodia semántica mixta y suele ser usado para expresar actitud negativa o neutral. Además, las actitudes neutrales expresadas ocupan un porcentaje relativamente alto porque demasiado se utiliza a menudo para expresar eufemismo en oraciones negativas, en las que la negación neutraliza el significado favorable o desfavorable de la palabra combinada. Por otro lado, en las oraciones afirmativas que expresan una actitud positiva, los adjetivos o adverbios con significado favorable que se combinan con 太(tài) en chino son más variados.

Cuando impartimos el español a alumnos chinos, la enseñanza asistida por el estudio de la prosodia semántica contribuye a la profundización del análisis semántico de la palabra al igual que evita o reduce la transferencia negativa de la lengua materna, debido a que en ciertos contextos es inadecuada la traducción mutua entre el demasiado y el 太(tài). Finalmente, consideramos que la visualización de las combinaciones más frecuentes de demasiado a través de la nube de palabras permite que la explicación de los vocablos por parte del profesorado sea más vívida y que la memorización de los usos por parte de los alumnos sea más fácil y rápida.

\section{Referencias}

Academia China de Ciencias Sociales. (2016). 现代汉语词典(第七版) xiàn dài hàn yǔ cí diăn (dì qī băn) [Diccionario de la lengua china moderna] (versión 7). Prensa Comercial (shāng wù yìn shū guăn). 
Anthony, L. (2020). AntConc (3.5.8) [Software]. AntLab. http://www.laurenceanthony.net/software.html

Berber, T. (2000). Semantic Prosodies in English and Portuguese: A Contrastive study. Cuadernos de Filología Inglesa, 9(01), 93-110. https://revistas. um.es/cfi/article/view/66701

Chen, X.-R. (2012). 词汇的语义韵及其翻译——基于 truly exceptional 汉译的个案 研究 cí huì de yǔ yì yùn jí qí fān yì——jī yú "truly exceptional" hàn yì de gè àn yán jiū [La prosodia semántica de los vocablos y su aplicación en la traducción: el caso de "truly exceptional" y su traducción al chino]. 当代外语研究 dāng dài wài yǔ yán jiū [Estudio Contemporáneo de Lenguas Extranjeras], (6), 24-27.

https://kns.cnki.net/kcms/detail/detail.aspx?dbcode=CJFD\&dbname=CJFD2 012\&filename $=$ KJYY201206008\&uniplatform $=$ NZKPT\&v=TWt2eAKk4K q0UWW4deMRl7FIyWxtSgyESGkx82EKCxSY6N5DVrqVcYQyGzzRfTsT Ciyun Ltd. (2020). Ciyunwenzi [Software online]. http://www.ciyunwenzi.com Emurasoft, Inc. (2012). EmEditor Profesional (12.0.0) [Software]. https:// www.emeditor.com

Ji, Y.-H., \& Wu, J.-P. (2003). 语义韵研究: 对象、方法及应用 yǔ yì yùn yán jiū: duì xiàng, fāng fă jí yìng yòng [Investigación sobre la prosodia semántica: objetos de estudio, métodos y aplicación]. 厦门大学学报(哲学社会科学版) xià mén dà xué xué bào (zhé xué shè huì kē xué băn) [Periódico Académico de la Universidad de Xiamen (Ciencias sociales y estudios filosóficos)], (3), 63-68. https://kns.cnki.net/kcms/detail/detail.aspx?dbcode $=$ CJFD\&dbname $=$ CJF D2000\&filename $=X M D S 200003009 \&$ uniplatform $=$ NZKPT\&v $=Z 1 \mathrm{kJp}$ \%25mmd2B8q16g3qAYQ1\%25mmd2FxP0x5DIxrGuE50jy3Rk5lc5ETO \%25mmd2FBp\%25mmd2F07dS5gWmRShFRdCR

$\mathrm{Li}, \mathrm{T}$. (2013). 基于语料库的汉英语义韵对比研究——以contribute to 为例jī yú yŭ liào kù de hàn yīng yǔ yì yùn duì bǐ yán jiū_—yǐ "contribute to" wèi lì [Comparación de la prosodia semántica entre chino e inglés basada en el corpus: el caso de “contribute to"]. 语文学刊 yǔ wén xué kān [Revista Académica de Filología China], (8), 16-18.

https://kns.cnki.net/kcms/detail/detail.aspx?dbcode $=$ CJFD\&dbname=CJF D2013\&filename $=$ YWWY201308009\&uniplatform $=$ NZKPT\&v=RfOql 9eVg\%25mmd2Bk7m8O8FVYxzSAJX1bjgy7v2R9s0Tg7meQILPfiV8Yc D\%25mmd2Fto\%25mmd2BDc8cUAX

Li, X.-H., \& Wei, N.-X. (2012). 汉英对应词语单位的语义趋向及语义韵对比研究 hàn yīng duì yìng cí yǔ dān wèi de yǔ yì qū xiàng jí yǔ yì yùn duì bǐ yán jiū [Estudio 
comparativo de la tendencia semántica y la prosodia semántica de las unidades lingüísticas entre chino e inglés]. 外语教学与研究 wài yǔ jiào xué yǔ yán jiū [Enseñanza y Estudio de Lenguas Extranjeras], 44(01), 20-33+157. https:// kns.cnki.net $/ \mathrm{kcms} /$ detail/detail.aspx?dbcode $=C J F D \& d b n a m e=C J F D 2012 \&$ filename $=$ WJYY201201005\&uniplatform $=$ NZKPT\&v $=6$ Yz76lnZOqoUmY6 MDKS\%25mmd2FSqalzEl9irO2FIHOk1V5P5VINWSwlJya4XvjXeTZQwtx Louw, B. (1993). Irony in the Text or Insincerity in the Writer? The Diagnostic Potential of Semantic Prosodies. En M. Baker, G. Francis \& E. Tognini-Bonelli (Eds.), Text and Technology: In Honour of John Sinclair (pp. 157-175). John Benjamins Publishing Company. https://doi. org/10.1075/z.64.11lou.

Ministerio de Educación. (2020). Corpus del chino moderno del Comité de Lenguas de China: CNCORPUS. Laboratorio de Lingüística Computacional, Instituto de Lingüística Aplicada, Ministerio de Educación. http://corpus. zhonghuayuwen.org/

Real Academia Española. (2020). Corpus del Español del Siglo XXI: CORPES XXI (versión 0.92). https://www.rae.es/banco-de-datos/corpes-xxi

Real Academia Española. (2021). Demasiado. En Diccionario de la lengua española. Consultado el 15 de septiembre de 2021. https://dle.rae.es/demasiado Schmid, H. (2019). Tree Tagger (3.0) [Software]. https://cis.uni-muenchen. $\mathrm{de} / \sim \mathrm{schmid} /$ tools/TreeTagger/

Shen, Y., \& Yang, L. (2019). 基于语料库的英汉近义词语义韵对比研究——以ANNOUN$C E, D E C L A R E$ 和宣布为例 jī yú yǔ liào kù de yīng hàn jìn yì cí yǔ yì yùn duì bǐ yán jiū yǐ ANNOUNCE, DECLARE hé "xuān bù" wèi lì [Estudio comparativo de la prosodia semántica de los sinónimos entre chino e inglés: el caso de “announce", “declare” y “xuān bù”]. 巢湖学院学报 cháo hú xué yuàn xué bào [Periódico Académico del Colegio Chaohu], 21(05), 120-126. https://kns.cnki. net/kcms/detail/detail.aspx?dbcode=CJFD\&dbname=CJFDLAST2020\&fil ename $=C H X Y 201905018$ \&uniplatform $=$ NZKPT\&v=wEJsoki744KSyVAlH 0RKUkJph0xK0F6tJPQmmhlCTc3QRDHxae8fV47vfOKs\%25mmd2Fp0J Sinclair, J. M. (1991). Corpus, Concordance, Collocation. Oxford University Press. Stubbs, M. (1996). Text and Corpus Analysis. Blackwell Publishers.

Tognini-Bonelli, E. (2002). Functionally complete units of meaning across English and Italian: Towards a corpus-driven approach. En B. Altenberg \& S. Granger (Eds.), Lexis in Contrast: Corpus-based Approaches (pp. 7495). John Benjamins Publishing Company. https://doi.org/10.1075/ scl.7.07tog 
Tong, F.-Z., \& Xiu, G. (2020). 基于语料库的中日对应词语义韵对比研究一一以中央文献日 译「堅持（する）」为例 jī yú yǔ liào kù de zhōng rì duì yìng cí yǔ yì yùn duì bǐ yán jiū——yì zhōng yāng wén xiàn rì yì 「jiān chí (する)」 wèi lì [Estudio comparativo de la prosodia semántica entre chino y japonés: el caso "jiān chî" y su traducción al japonés “する” en los documentos oficiales del gobierno central]. 日语学习与研究 rì yŭ xué xí yǔ yán jiū [Aprendizaje y Estudio del Japonés], (02), 1-8.

https://kns.cnki.net/kcms/detail/detail.aspx?dbcode $=$ CJFD\&dbname $=$ CJF DLAST2020\&filename $=$ RYXY202002001\&uniplatform $=\mathrm{NZKPT \& v}=1 \mathrm{Z}$ 8JAoJJ9GeMtFadzeXsDs3tURvx8Qz1IsRaFX22oUozcC\%25mmd2Bza SnqBzfijJ54e\%25mmd2FPz

Wei, N.-X. (2002). 语义韵研究的一般方法 yǔ yì yùn yán jiū de yì bān fāng fă [Los métodos generales para investigar la prosodia semántica]. 外语教学与研究 wài yǔ jiào xué yǔ yán jiū [Enseñanza y Estudio de Lenguas Extranjeras], (04), 300-307.

https://kns.cnki.net/kcms/detail/detail.aspx?dbcode=CJFD\&dbname=CJF D2002\&filename $=$ WJYY200204010\&uniplatform $=$ NZKPT\&v=PiIALvj vnze510\%25mmd2B5aOYTVPu58SIrzYpLsnZs3OFRApngZqwA98k9x wPiZlXO6fuY

You, H.-J. (2011). 基于语料库的近义词辨析 jĩ yú yǔ liào kù de jìn yì cí biàn xī [La distinción de los sinónimos basada en el corpus]. 语文学刊 yǔ wén xué kān [Revista Académica de Filología China], (01), 91-92. https://www. taodocs.com/p-347383142.html

Zhao, X.-L., \& Li, S.-J. (2010). 语料库驱动的跨语言语义韵研究——以“too” 和“太”的语义韵对比为例 yǔ liào kù qū dòng de kuà yǔ yán yǔ yì yùn yán jiū-—yǐ " too " hé " tài " de yǔ yì yùn duì bǐ wèi lì [Estudio intralingüístico de la prosodia semántica impulsado por el corpus: el contraste de “too” y “Tài”]. 现代语文(语言研究版) xiàn dài yǔ wén( yǔ yán yán jiū băn) [Filología China Moderna (Estudios de lenguas)], (12), 78-80. https:// kns.cnki.net $/ \mathrm{kcms} /$ detail/detail.aspx?dbcode $=C J F D \& d b n a m e=C J F D L A$ SN2019\&filename $=Y W L Y 201012032 \&$ uniplatform $=N Z K P T \& v=0 p u 88 j$ mOsmFDkvmol0n\%25mmd2B1fECA0fiJp8Q7XKlIqPdOoGYreX6QwAi Ci\%25mmd2FkNnXjVSrH

Zheng, S.-J. (2020). Prólogo. In Cao, Y.-F. (Ed.), 语言学导论 yǔ yán xué dăo lùn [Introducción a la lingüística] (pp. 1-3). 北京: 外语教学与研究出版 社 běi jīng : wài yǔ jiào xué yǔ yán jiū chū băn shè [Beijing: Editorial de Enseñanza y Estudios de Lenguas Extranjeras]. 